\title{
Evidence for unidirectional nematic bond ordering in FeSe
}

\author{
M. D. Watson, ${ }^{1, *}$ T. K. Kim, ${ }^{1}$ L. C. Rhodes, ${ }^{2}$ M. Eschrig, ${ }^{2}$ M. Hoesch, ${ }^{1}$ A. A. Haghighirad, ${ }^{3}$ and A. I. Coldea ${ }^{3}$ \\ ${ }^{1}$ Diamond Light Source, Harwell Campus, Didcot OX11 ODE, United Kingdom \\ ${ }^{2}$ Department of Physics, Royal Holloway, University of London, Egham, Surrey TW20 OEX, United Kingdom \\ ${ }^{3}$ Clarendon Laboratory, Department of Physics, University of Oxford, Parks Road, Oxford OX1 3PU, United Kingdom
}

(Received 8 April 2016; revised manuscript received 2 October 2016; published 11 November 2016)

\begin{abstract}
The presence of $d_{x z}-d_{y z}$ orbital ordering is often considered a hallmark of the nematic phase of Fe-based superconductors, including FeSe, but the details of the order parameter remain controversial. Here, we report a high-resolution angle-resolved photoemission spectroscopy study of single crystals of FeSe, accounting for the photon-energy dependence and making a detailed analysis of the temperature dependence. We find that the hole pocket undergoes a fourfold-symmetry-breaking distortion in the nematic phase below $90 \mathrm{~K}$, but, in contrast, the changes to the electron pockets do not require fourfold symmetry breaking. Instead, there is an additional separation of the existing $d_{x y}$ and $d_{x z / y z}$ bands, which themselves are not split within resolution. These observations lead us to propose a scenario of "unidirectional nematic bond ordering" to describe the low-temperature electronic structure of FeSe, supported by good agreement with ten-orbital tight-binding model calculations.
\end{abstract}

DOI: 10.1103/PhysRevB.94.201107

The search for the understanding of unconventional superconductivity in the Fe-based systems has led to a focus on the origin and nature of the ordered phases found in close proximity to the superconducting phase. Particular attention has been drawn to the "nematic" phase [1], where the fourfold symmetry of the lattice is broken at a temperature $T_{s}$ which is higher than the striped antiferromagnetic ordering temperature $T_{N}$ in some materials [2]. There has been long discussion about whether these two transitions are both magnetic in origin, or whether $T_{s}$ corresponds to a separate orbital instability [1]. FeSe is an exceptional case, since it undergoes a structural transition at $T_{s}=90 \mathrm{~K}$ without longrange magnetic order at any temperature, enabling detailed studies of the symmetry-broken state [3-9], and has therefore attracted much theoretical attention [10-16]. Angle-resolved photoemission spectroscopy (ARPES) measurements of FeSe [17-28] provide direct experimental access to the evolution of the electronic structure through $T_{s}$, which can be linked to theoretical models of the underlying symmetry-breaking order.

Previous ARPES studies of FeSe have consistently found a quasi-two-dimensional (2D) electronic structure with holelike bands around the $\Gamma$ point and electronlike bands around the $M$ point. The Fermi surfaces are found to be significantly smaller than expected from density functional theory calculations (but consistent with quantum oscillations [21]), and moderately strong orbital-dependent band renormalizations are found $[18,21]$. However, the understanding of the temperature dependence of the ARPES spectra, which has direct relevance to the identification of the nematic order parameter, has not yet been firmly established [29]. Most previous ARPES studies

\footnotetext{
*Author to whom correspondence should be addressed: matthew.watson@diamond.ac.uk

Published by the American Physical Society under the terms of the Creative Commons Attribution 3.0 License. Further distribution of this work must maintain attribution to the author(s) and the published article's title, journal citation, and DOI.
}

have paid particular attention to the electron pockets around the $M$ point, and several inferred a $\sim 50 \mathrm{meV}$ splitting of $d_{x z}$ and $d_{y z}$ bands [19-24,26,28], similar to previous claims in $\mathrm{NaFeAs}$ [30] and $\mathrm{Ba}\left(\mathrm{Fe}_{1-x} \mathrm{Co}_{x}\right)_{2} \mathrm{As}_{2}$ [31]. In this scenario, which could be interpreted as a ferro-orbital ordering [21], the bands of primarily $d_{x z}$ and $d_{y z}$ character are degenerate exactly at $M$ in the high-temperature phase [32], but split below $T_{s}$. Then, the two main features at the $M$ point observed by ARPES at low temperature both arise from $d_{x z / y z}$ bands, with the assumption that the outer electron band with $d_{x y}$ character does not contribute [21,24]. Numerous theoretical alternatives to the ferro-orbital ordering scenario have also been recently proposed [10,24,33-35], but a $d_{x z / y z}$ splitting at the $M$ point has always previously been considered as a hallmark of the nematic phase.

In this Rapid Communication, we present a high-resolution ARPES study of the evolution of the electronic structure of bulk FeSe through the nematic transition at $T_{s}$. We use curvature analysis and a procedure to fit the energy dispersion curves (EDCs) at the $M$ point to extract band positions. We also observe the $k_{z}$ dependence of the electron pockets, and present Fermi surface maps covering the whole Brillouin zone above and below $T_{s}$. While the hole pockets of FeSe do undergo significant symmetry-breaking distortions below $T_{s}$, the changes to the electron pockets arise from an additional separation of existing $d_{x z / y z}$ and $d_{x y}$ bands, without any resolvable lifting of $d_{x z}-d_{y z}$ band degeneracy at the $M$ point. These observations exclude the ferro-orbital scenario, and place strong constraints on the nature of the orbital ordering. Finally, we use a ten-orbital tight-binding model to propose that the nematic state of FeSe is characterized by rotationalsymmetry breaking within Fe-Fe hoppings, which may be described as a unidirectional nematic bond ordering.

High-quality single crystal samples of FeSe were grown by the chemical vapor transport method [3,21,36]. ARPES measurements were performed at the I05 beamline at the Diamond Light Source, U.K.

In Figs. 1(a)-1(d) we present temperature-dependent ARPES data for a high-symmetry cut centered on the $M$ 

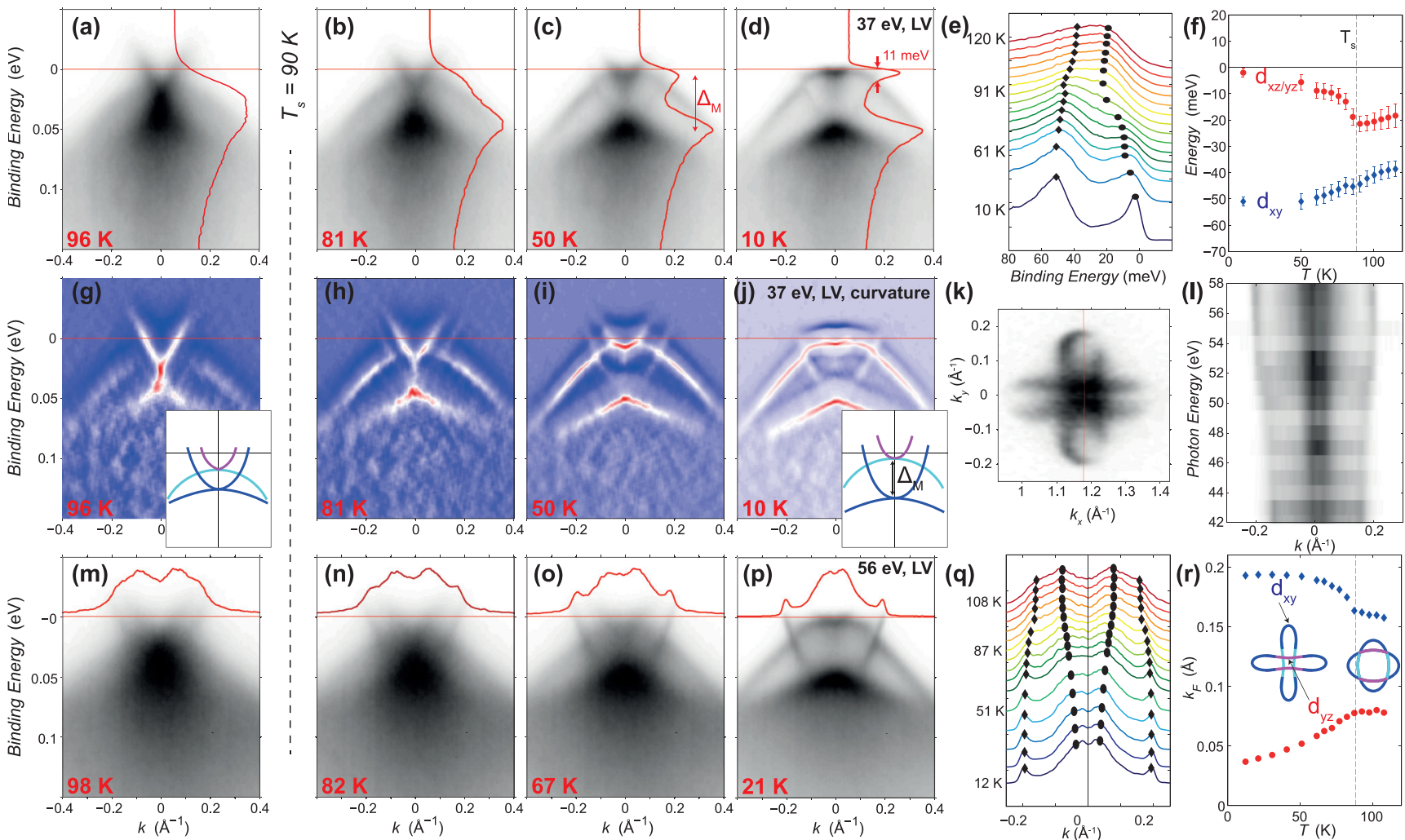

FIG. 1. (a)-(d) ARPES intensity plots of a high-symmetry cut through the $M$ point, using $37 \mathrm{eV}$ photon energy in vertical polarization ( $L V$, perpendicular to the scattering plane). Solid lines plot the EDC at $M$, which are also stacked according to temperature in (e). (f) Temperature dependence of peak positions extracted from the EDC analysis described in the text. (g)-(j) Curvature plots, showing traces of all expected bands (as shown in cartoon insets) above and below $T_{s}$. (k) Low-temperature electron Fermi surface at 56 eV. (l) Photon-energy dependence of the momentum distribution curve (MDC) through the $M$ point at $10 \mathrm{~K}$, which corresponds to the $k_{z}$ dispersion of the electron pocket. The A point where pockets are largest corresponds to $56 \mathrm{eV}$. (m)-(p) High-symmetry cuts [along the red line in $(\mathrm{k})$ ] through the A point at $56 \mathrm{eV}$, showing also the MDC at the Fermi level. (q) MDCs plotted as a function of temperature and (r) temperature dependence of $k_{F}$ vectors extracted from fits to MDCs.

point, also showing the EDCs at $M$. At low temperatures [Fig. 1(d)], these EDCs display two prominent peaks with a separation $\Delta_{M}=50 \mathrm{meV}$, often previously attributed to orbital splitting in the nematic phase, i.e., $\Delta_{M}^{F O}=E_{y z}-E_{x z}$. However, curvature analysis [37] enhances weak features in the data and provides a different perspective, as shown in Figs. 1(g)-1(j). Above $T_{s}$, in Fig. $1(\mathrm{~g})$ both the expected $d_{x z}$ and $d_{y z}$ dispersions and crucially also sections with $d_{x y}$ character are observed. By comparing Figs. 1(g)-1(j), we observe the similarity in the dispersions above and below $T_{s}$, and no extra features arise. Therefore, here we propose a scenario where the low-temperature dispersions simply consist of the $d_{x z / y z}$ and $d_{x y}$ bands, as expected above $T_{s}$, but with an increased separation between them. We assign the peaks in the EDCs to the two bands, i.e., $\Delta_{M}=E_{x z / y z}-E_{x y}$, equal to $50 \mathrm{meV}$ at low temperatures but also finite above $T_{s}$. Within experimental resolution $[11 \mathrm{meV}$ full width at half maximum (FWHM) for the relevant peak in Fig. 1(d)] we do not detect any subsequent splitting between $d_{x z}$ and $d_{y z}$ bands, although since this is allowed by symmetry in the orthorhombic phase, a small splitting may occur as a secondary effect.

This scenario can be tested by the extraction of band positions as a function of temperature from the EDCs at the $M$ point. However, while the separation $\Delta_{M}$ of the two features in the EDC is unambiguous at low temperatures, it becomes more difficult to define at higher temperatures, as features become broader. Here, we take a different approach: We fit the EDC with two asymmetric pseudo-Voigt functions and the Fermi function at $61 \mathrm{~K}$ where the peaks are still distinct. Then, at higher temperatures, we fit using the same peak profiles, only allowing the peak positions to vary [see the Supplemental Material (SM) [38]]. We find that the fitted peak positions are separated by $20 \mathrm{meV}$ even above $T_{s}$, but increase substantially when the system enters the nematic phase [Figs. 1(e) and 1(f)]. The sharp upward shift of the $d_{x z / y z}$ bands at $T_{s}$ is the strongest feature, although the $d_{x y}$ band position also adjusts downwards below $T_{s}$, leading to a total separation of $50 \mathrm{meV}$ at $10 \mathrm{~K}$.

In Figs. 1(m)-1(p) we present measurements of the electron pockets using a photon energy of $56 \mathrm{eV}$, where the outer $d_{x y}$ electron dispersion is already clearly visible in the data above $T_{s}$ even without curvature analysis. The $56 \mathrm{eV}$ photon energy is chosen to correspond to the A point at the top of the Brillouin zone where the warped quasi-2D electron pocket is largest, giving the best momentum resolution of features, as can be seen in Fig. 1(1). Further details of the photon-energy dependence are presented in SM, where it is also shown that $\Delta_{M}$ is independent of $k_{z}$. In Figs. 1(q) and 1(r) we extract the temperature dependence of both $k_{F}$ vectors observed, 
(a) $\mathrm{hv}=56 \mathrm{eV}, \mathrm{LV} 100 \mathrm{~K}$

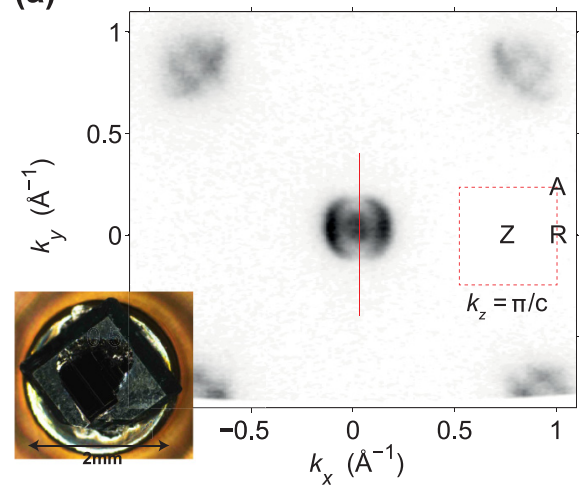

(d)

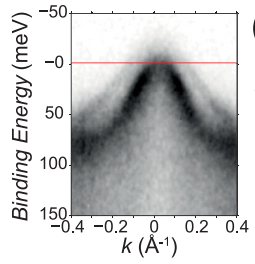

(b)

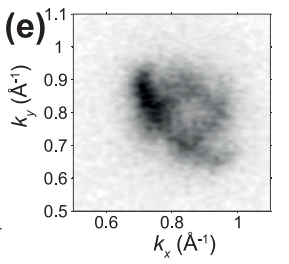

(f)
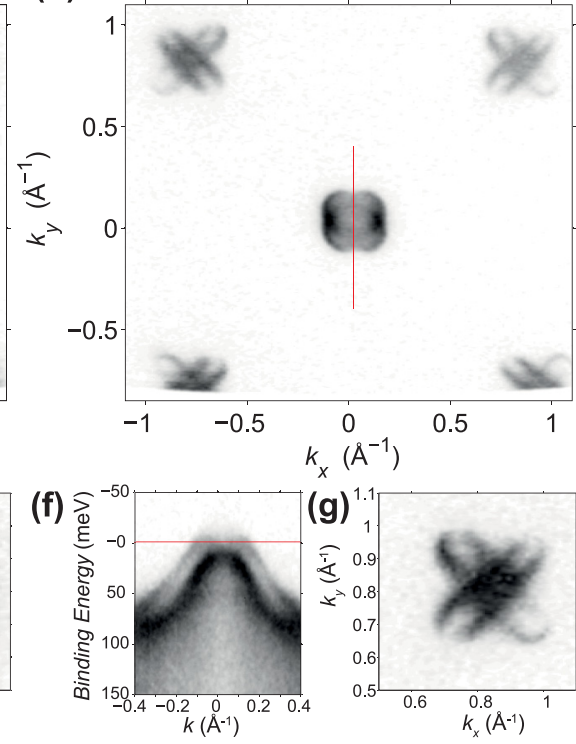

$50 \mathrm{~K}$

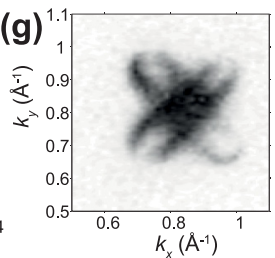

(c)

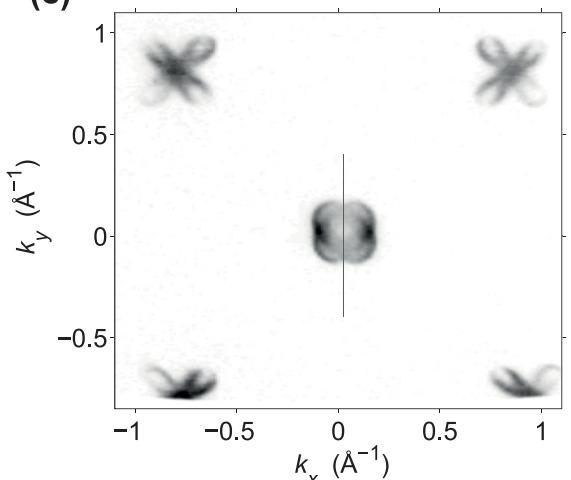

(h)

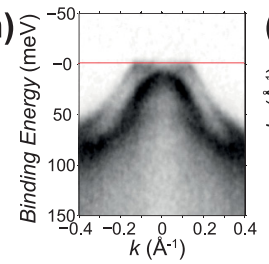

$7 \mathrm{~K}$

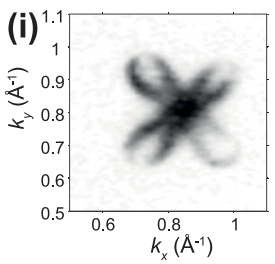

FIG. 2. (a)-(c) Temperature-dependent Fermi surface maps of FeSe near the top of the Brillouin zone, taken using $56 \mathrm{eV}$ photon energy in $L V$ polarization and integrating spectral weight within $2 \mathrm{meV}$ of the Fermi level. Inset to (a) is the sample used. (d), (f), (h) Cuts through the $Z$ point $[R Z R$ direction: solid red line in maps (a)-(c)], revealing the extra splitting which pushes the inner hole band below the Fermi level. (e), (g), (i) Expanded Fermi surface map of the electron pocket: Both the inner and outer electron bands are clearly detected.

from constrained Lorentzian peak fitting of the momentum distribution curve (MDC) at the Fermi level (SM). The $k_{F}$ values are well defined at all temperatures and also demonstrate that the $d_{y z}$ and $d_{x y}$ dispersions are separate above $T_{s}$ and undergo additional separation below $T_{s}$.

In Fig. 2 we present further support for this interpretation, including the observation of the $d_{x y}$ electron band. These ARPES data are obtained with the scattering plane at $45^{\circ}$ to the Fe square lattice to mitigate matrix element effects [39], again using $56 \mathrm{eV}$ photon energy. At $100 \mathrm{~K}$, in the tetragonal phase above $T_{s}$, the Fermi surface maps [Figs. 2(a) and 2(e)] reveal essentially the whole structure of the expected electron pockets at the A point, including the outer pocket with $d_{x y}$ character. Since all expected bands contribute at high temperature, we conclude that the whole structure of the low-temperature Fermi surface is also observed. The comparison between Figs. 2(e) and 2(i) demonstrates that the low-temperature Fermi surface is elongated compared to the high-temperature case, but retains the same basic structure and symmetry. Our samples are twinned, but since the electron pockets retain fourfold symmetry, the bands from both structural domains will overlap in experiments. Future high-resolution detwinned measurements may be able to confirm this scenario.

Recently, it has been separately argued in two other ARPES studies $[27,28]$ that the $d_{x y}$ bands do contribute to the spectra at the $M$ point, consistent with our analysis. Both groups also claimed that the $d_{x z / y z}$ bands split in the nematic phase, and that either three [28] or four [27] separate band dispersions are present at the $M$ point. However, we do not find any evidence for more than two features in the MDC or EDC or curvature analysis at the $M$ point in any measurement condition. The preservation of $d_{x z / y z}$ degeneracy at $M$ (to within experimental resolution) is what distinguishes our interpretation from other proposals.
The evolution of the electron pockets through $T_{s}$ contrasts strongly with the behavior of the hole pockets. At the $Z$ point [Fig. 2(d)], above $T_{s}$ the inner hole pocket just crosses the Fermi level, making a small three-dimensional (3D) pocket [21,23], and there is a $\sim 20 \mathrm{meV}$ splitting due to spin-orbit coupling at the $Z$ point between the $d_{x z / y z}$ bands. However, below $T_{s}$ there is an extra splitting of $\sim 15 \mathrm{meV}$ associated with a $d_{x z / y z}$ orbital polarization, in addition to the spin-orbit splitting. This pushes the inner band completely below the Fermi level [Fig. 2(h)]. Therefore, there is a single hole band at low temperatures which displays an elliptical distortion, however, due to the presence of twin domains below $T_{s}$, two crossed ellipses are superposed in the ARPES data in Figs. 2(b) and 2(c).

The breaking of fourfold rotational symmetry of the hole pockets below $T_{s}$ is therefore well established. However, we have found symmetry-preserving changes to the electronic structure at the $M$ point, which on first sight are difficult to reconcile with the tetragonal-to-orthorhombic phase transition at $T_{s}$. Another constraint is that backfolded bands are not observed, suggesting that translational symmetry is preserved at $T_{s}$. The challenge is to identify an orbital order parameter which globally breaks fourfold rotational symmetry but is consistent with the strong constraints provided by these observations. Ferro-orbital ordering could account for the symmetry breaking at the $\Gamma$ point, but requires a splitting of $d_{x z / y z}$ orbitals at the $M$ point, which we have argued is not the case (see also SM). Moreover, ferro-orbital ordering is not consistent with the direction of distortion of the hole band, as revealed by ARPES measurements on detwinned crystals [24]. A Néel-type antiferro-orbital ordering would preserve the $d_{x z / y z}$ degeneracy at $M$, but it cannot explain the extra splitting observed between $d_{x z / y z}$ bands at the $\Gamma / Z$ point [33]. The recently proposed $d$-wave bond nematic order predicts 


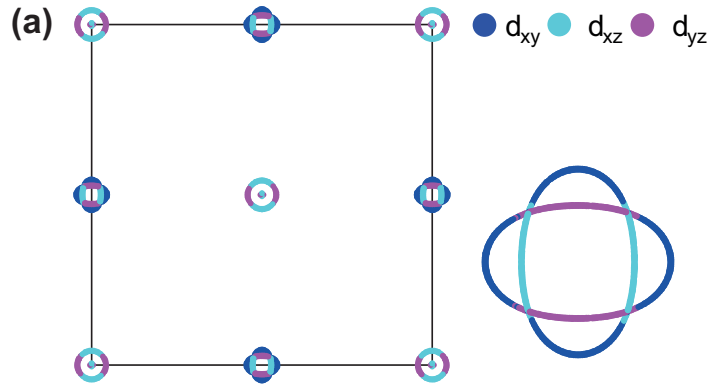

(b)

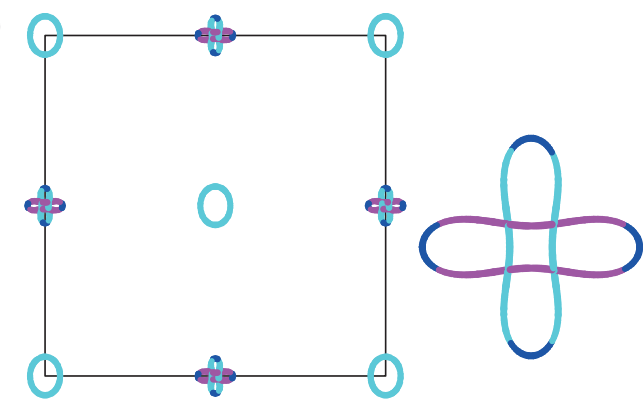

(c)

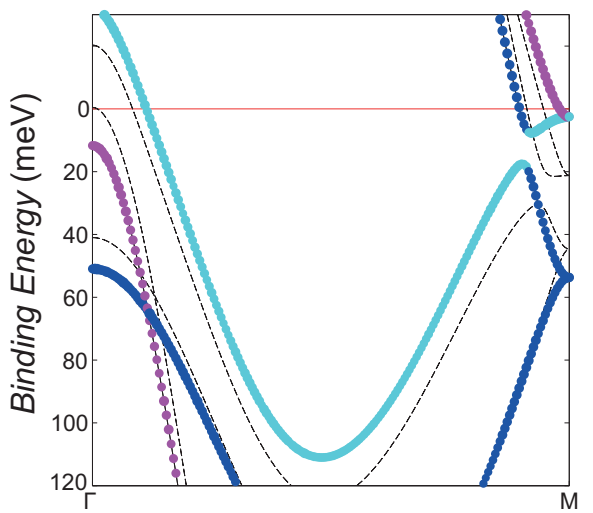

FIG. 3. (a) Fermi surface calculated using a ten-orbital tightbinding model for FeSe in the tetragonal phase, showing the folded band structure, and (b) with the unidirectional nematic bond order parameter, which distorts the hole pocket but preserves fourfold symmetry of the electron pocket. (c) Band structure along $\Gamma-M$ $[(0,0)-(0, \pi)$ direction] with the dashed lines corresponding to the tetragonal phase. Note the extra splitting at the $\Gamma$ point and the symmetric shift of $d_{x z / y z}$ bands at the $M$ point.

a splitting at the $M$ point $[33,34]$, as does the microscopic model of Ref. [35]. However, we suggest that a "unidirectional nematic bond ordering" is compatible with all the observations.

In Fig. 3 we present tight-binding model electronic structures with and without the proposed unidirectional nematic bond order. We use a 2D ten-orbital tight-binding model including spin-orbit coupling $[13,40]$, with hopping parameters adjusted to match the high-temperature band structure (SM). Within this model, we add an order parameter to the intersite hopping block of the Hamiltonian as $h=\Delta_{S}\left(n_{y z}^{\prime}-\right.$ $\left.n_{x z}^{\prime}\right) \cos \left(k_{x}\right)$, where $n_{x z}^{\prime}$ indicates the intersite hopping operator distinct from on-site occupations $n_{x z}$. This order parameter therefore describes a symmetry breaking within the intersite $d_{x z / y z}$ hopping terms on the Fe-Fe bonds in the $x$ direction. This order parameter has the desired properties of giving an extra splitting in addition to the spin-orbit coupling splitting of $d_{x z / y z}$ bands at the $\Gamma$ point, but a symmetric shift up of the $d_{x z / y z}$ bands at the $M$ point without losing the degeneracy. It still globally breaks fourfold rotational symmetry, yet it can also be simply shown that it does not break translational symmetry (SM). In Figs. 3(b) and 3(c) we present the results of a calculation with $\Delta_{S}=20 \mathrm{meV}$; we also include an adjustment of $-10 \mathrm{meV}$ to the $d_{x y}$ orbital which is motivated by the experimental results in Fig. 1 and may be required to maintain the charge balance of the system. Within this fairly simple model we obtain Fermi surfaces and dispersions which reproduce all qualitative features of the low-lying electronic structure. Therefore, we suggest that the changes to the electronic structure of $\mathrm{FeSe}$ in the orthorhombic phase may be primarily described by a unidirectional nematic bond ordering.

Since the Fermi surface changes at the $M$ point are not of a symmetry-breaking nature, and $\Delta_{M}$ is finite even above $T_{s}$, there is an important question about how tightly the evolution of the electron pockets is linked to $T_{s}$. However, we have shown that there is a sharp increase in $\Delta_{M}$ which onsets exactly at $T_{s}$ [Fig. 1(j)]. Additionally, the deviation in $k_{F}$ values of both the inner and outer electron pocket branches also follows a sharp order-parameter-like behavior which onsets at $T_{s}$, and this deviation of the inner pocket was shown to behave as an order parameter of the structural transition across the $\mathrm{FeSe}_{1-x} \mathrm{~S}_{x}$ series [23]. This indicates that the changes at the $M$ point are still fundamentally linked to the orthorhombic lattice distortion. This may be understood since in the unfolded 1-Fe Brillouin zone, the electron pockets with $d_{x z}$ and $d_{y z}$ character are located in different parts of $k$ space, and therefore the $\cos \left(k_{x}\right)$ term ensures that they shift symmetrically, such that degeneracy is not lost in the folded 2-Fe zone (SM). Therefore, the apparently non-symmetrybreaking band movements at $M$ are linked to an ordering which globally does break tetragonal symmetry, concomitant with the tetragonal-orthorhombic lattice distortion at $T_{s}$.

Within the longstanding debate about the roles of orbital and spin degrees of freedom in Fe-based superconductors [1], FeSe is often considered as an example where orbital interactions may be dominant $[3-5,21]$. However, we have excluded any significant ferro-orbital ordering, and, furthermore, we have found that the primary order parameter is bond centered and that translational symmetry is not broken, in contrast to some proposals $[10,41]$. Intriguingly, a bond-centered ordering is also observed in cuprates [42], although in that case it has an incommensurate modulation. On the other hand, there are mixed reports on how relevant magnetic interactions are to the structural transition in FeSe [3-8,11]. The magnitude of energy shifts (e.g., $20 \mathrm{meV}$ for the $d_{x z / y z}$ bands at $M$ ) are similar to the spin-orbit coupling value of $20 \mathrm{meV}$ in FeSe [21], suggesting that a spin-driven scenario is not ruled out. Thus our data exclude several proposed orbital ordering scenarios, and instead point to the existence of a unidirectional nematic bond ordered phase in FeSe, breaking rotational but not translational symmetry, which is distinct from the known striped antiferromagnetic phase in other Fe-based superconductors.

In conclusion, we have presented a high-resolution ARPES study of FeSe, and argued that the symmetry-breaking distortion of the hole pockets at the $\Gamma$ point but symmetry-preserving 
changes of the electron pockets at the $M$ point below $T_{s}$ point can be explained by a unidirectional nematic bond ordering. These measurements provide a fresh perspective on the nature of the nematic phase of Fe-based superconductors.

We thank T. Scaffidi, R. Valenti, M. Rahn, Z. Wang, R. Fernandes, and F. Kruger for useful discussions. We thank N. B. M. Schröter, L. B. Duffy, S. F. Blake, and A. Narayanan for technical assistance. We thank Diamond Light Source for access to Beamline I05 (Proposals No.
SI10203, No. SI11792, and No. SI12799) that contributed to the results presented here. Part of the work was supported by the EPSRC (EP/L001772/1, EP/I004475/1, EP/I017836/1). A.A.H. acknowledges the financial support of the Oxford Quantum Materials Platform Grant (EP/M020517/1). A.I.C. acknowledges an EPSRC Career Acceleration Fellowship (Grant No. EP/I004475/1).

In accordance with the EPSRC policy framework on research data, access to the data will be made available from http://dx.doi.org/10.5287/bodleian:BevPZ676B.
[1] R. M. Fernandes, A. V. Chubukov, and J. Schmalian, Nat. Phys. 10, 97 (2014).

[2] M. G. Kim, R. M. Fernandes, A. Kreyssig, J. W. Kim, A. Thaler, S. L. Bud'ko, P. C. Canfield, R. J. McQueeney, J. Schmalian, and A. I. Goldman, Phys. Rev. B 83, 134522 (2011).

[3] A. E. Böhmer, F. Hardy, F. Eilers, D. Ernst, P. Adelmann, P. Schweiss, T. Wolf, and C. Meingast, Phys. Rev. B 87, 180505 (2013).

[4] A. E. Böhmer, T. Arai, F. Hardy, T. Hattori, T. Iye, T. Wolf, H. v. Löhneysen, K. Ishida, and C. Meingast, Phys. Rev. Lett. 114, 027001 (2015).

[5] S.-H. Baek, D. V. Efremov, J. M. Ok, J. S. Kim, J. van den Brink, and B. Büchner, Nat. Mater. 14, 210 (2015).

[6] M. C. Rahn, R. A. Ewings, S. J. Sedlmaier, S. J. Clarke, and A. T. Boothroyd, Phys. Rev. B 91, 180501 (2015).

[7] Q. Wang, Y. Shen, B. Pan, Y. Hao, M. Ma, F. Zhou, P. Steffens, K. Schmalzl, T. R. Forrest, M. Abdel-Hafiez, X. Chen, D. A. Chareev, A. N. Vasiliev, P. Bourges, Y. Sidis, H. Cao, and J. Zhao, Nat. Mater. 15, 159 (2016).

[8] Q. Wang, Y. Shen, B. Pan, X. Zhang, K. Ikeuchi, K. Iida, A. D. Christianson, H. C. Walker, D. T. Adroja, M. Abdel-Hafiez, X. Chen, D. A. Chareev, A. N. Vasiliev, and J. Zhao, Nat. Commun. 7, 12182 (2016).

[9] M. D. Watson, T. Yamashita, S. Kasahara, W. Knafo, M. Nardone, J. Béard, F. Hardy, A. McCollam, A. Narayanan, S. F. Blake, T. Wolf, A. A. Haghighirad, C. Meingast, A. J. Schofield, H. v. Löhneysen, Y. Matsuda, A. I. Coldea, and T. Shibauchi, Phys. Rev. Lett. 115, 027006 (2015).

[10] A. V. Chubukov, R. M. Fernandes, and J. Schmalian, Phys. Rev. B 91, 201105 (2015).

[11] J. K. Glasbrenner, I. I. Mazin, H. O. Jeschke, P. J. Hirschfeld, R. M. Fernandes, and R. Valentí, Nat. Phys. 11, 953 (2015).

[12] R. Yu and Q. Si, Phys. Rev. Lett. 115, 116401 (2015).

[13] S. Mukherjee, A. Kreisel, P. J. Hirschfeld, and B. M. Andersen, Phys. Rev. Lett. 115, 026402 (2015).

[14] Y. Yamakawa, S. Onari, and H. Kontani, Phys. Rev. X 6, 021032 (2016).

[15] F. Wang, S. A. Kivelson, and D.-H. Lee, Nat. Phys. 11, 959 (2015).

[16] S. W. Lovesey, Phys. Rev. B 93, 085126 (2016).

[17] S. Tan, Y. Zhang, M. Xia, Z. Ye, F. Chen, X. Xie, R. Peng, D. Xu, Q. Fan, H. Xu, J. Jiang, T. Zhang, X. Lai, T. Xiang, J. Hu, B. Xie, and D. Feng, Nat. Mater. 12, 634 (2013).

[18] J. Maletz, V. B. Zabolotnyy, D. V. Evtushinsky, S. Thirupathaiah, A. U. B. Wolter, L. Harnagea, A. N. Yaresko, A. N. Vasiliev, D. A. Chareev, A. E. Böhmer, F. Hardy, T. Wolf, C. Meingast, E.
D. L. Rienks, B. Büchner, and S. V. Borisenko, Phys. Rev. B 89, 220506 (2014).

[19] T. Shimojima, Y. Suzuki, T. Sonobe, A. Nakamura, M. Sakano, J. Omachi, K. Yoshioka, M. Kuwata-Gonokami, K. Ono, H. Kumigashira, A. E. Böhmer, F. Hardy, T. Wolf, C. Meingast, H. V. Löhneysen, H. Ikeda, and K. Ishizaka, Phys. Rev. B 90, 121111 (2014).

[20] K. Nakayama, Y. Miyata, G. N. Phan, T. Sato, Y. Tanabe, T. Urata, K. Tanigaki, and T. Takahashi, Phys. Rev. Lett. 113, 237001 (2014).

[21] M. D. Watson, T. K. Kim, A. A. Haghighirad, N. R. Davies, A. McCollam, A. Narayanan, S. F. Blake, Y. L. Chen, S. Ghannadzadeh, A. J. Schofield, M. Hoesch, C. Meingast, T. Wolf, and A. I. Coldea, Phys. Rev. B 91, 155106 (2015).

[22] P. Zhang, T. Qian, P. Richard, X. P. Wang, H. Miao, B. Q. Lv, B. B. Fu, T. Wolf, C. Meingast, X. X. Wu, Z. Q. Wang, J. P. Hu, and H. Ding, Phys. Rev. B 91, 214503 (2015).

[23] M. D. Watson, T. K. Kim, A. A. Haghighirad, S. F. Blake, N. R. Davies, M. Hoesch, T. Wolf, and A. I. Coldea, Phys. Rev. B 92, 121108 (2015).

[24] Y. Suzuki, T. Shimojima, T. Sonobe, A. Nakamura, M. Sakano, H. Tsuji, J. Omachi, K. Yoshioka, M. Kuwata-Gonokami, T. Watashige, R. Kobayashi, S. Kasahara, T. Shibauchi, Y. Matsuda, Y. Yamakawa, H. Kontani, and K. Ishizaka, Phys. Rev. B 92, 205117 (2015).

[25] S. V. Borisenko, D. V. Evtushinsky, Z.-H. Liu, I. Morozov, R. Kappenberger, S. Wurmehl, B. Buchner, A. N. Yaresko, T. K. Kim, M. Hoesch, T. Wolf, and N. D. Zhigadlo, Nat. Phys. 12, 311 (2016).

[26] W. Li, Y. Zhang, J. J. Lee, H. Ding, M. Yi, Z. Li, P. Deng, K. Chang, S. K. Mo, M. Hashimoto, D. H. Lu, X. Chen, R. G. Moore, Q. K. Xue, and Z. X. Shen, arXiv:1509.01892.

[27] A. Fedorov, A. Yaresko, T. K. Kim, E. Kushnirenko, E. Haubold, T. Wolf, M. Hoesch, A. Grueneis, B. Buechner, and S. V. Borisenko, arXiv:1606.03022.

[28] L. Fanfarillo, J. Mansart, P. Toulemonde, H. Cercellier, P. L. Fèvre, F. Bertran, B. Valenzuela, L. Benfatto, and V. Brouet, Phys. Rev. B 94, 155138 (2016).

[29] Y. V. Pustovit and A. A. Kordyuk, arXiv:1608.07751.

[30] M. Yi, D. H. Lu, R. G. Moore, K. Kihou, C.-H. Lee, A. Iyo, H. Eisaki, T. Yoshida, A. Fujimori, and Z.-X. Shen, New J. Phys. 14, 073019 (2012).

[31] M. Yi, D. Lu, J.-H. Chu, J. G. Analytis, A. P. Sorini, A. F. Kemper, and B. Moritz, Proc. Natl. Acad. Sci. USA 108, 6878 (2011).

[32] R. M. Fernandes and O. Vafek, Phys. Rev. B 90, 214514 (2014). 
[33] Y. Liang, X. Wu, and J. Hu, Chin. Phys. Lett. 32, 117402 (2015).

[34] K. Jiang, J. Hu, H. Ding, and Z. Wang, Phys. Rev. B 93, 115138 (2016).

[35] S. Onari, Y. Yamakawa, and H. Kontani, Phys. Rev. Lett. 116, 227001 (2016).

[36] D. Chareev, E. Osadchii, T. Kuzmicheva, J.-Y. Lin, S. Kuzmichev, O. Volkova, and A. Vasiliev, CrystEngComm 15, 1989 (2013).

[37] P. Zhang, P. Richard, T. Qian, Y. M. Xu, X. Dai, and H. Ding, Rev. Sci. Instrum. 82, 043712 (2011).

[38] See Supplemental Material at http://link.aps.org/supplemental/ 10.1103/PhysRevB.94.201107 for details of the fitting analysis of MDCs and EDCs, further photon-energy dependence, and consideration of alternative order parameters.

[39] V. Brouet, M. F. Jensen, P. H. Lin, A. Taleb-Ibrahimi, P. Le Fèvre, F. Bertran, C. H. Lin, W. Ku, A. Forget, and D. Colson, Phys. Rev. B 86, 075123 (2012).

[40] H. Eschrig and K. Koepernik, Phys. Rev. B 80, 104503 (2009).

[41] H. Kontani, T. Saito, and S. Onari, Phys. Rev. B 84, 024528 (2011).

[42] R. Comin, R. Sutarto, F. He, E. H. da Silva Neto, L. Chauviere, A. Frano, R. Liang, W. N. Hardy, D. A. Bonn, Y. Yoshida, H. Eisaki, A. J. Achkar, D. G. Hawthorn, B. Keimer, G. A. Sawatzky, and A. Damascelli, Nat. Mater. 14, 796 (2015). 